\title{
Dual appearance of fluorescein staining in vivo of diseased human corneal epithelium. A non-contact photomicrographic study
}

University of Lund, Department of Ophthalmology, Malmö, S-21401, Sweden HM Tabery

Correspondence to: Dr Helena M Tabery.

Accepted for publication 20 May 1991

\begin{abstract}
Adherence of fluorescein sodium dye to diseased epithelial cells, a hitherto unreported phenomenon, was captured in photomicrographs in severe herpes zoster and keratoconjunctivitis sicca keratopathies. It is notable that this phenomenon differs completely from the well known fluorescent property of the dye penetrating into defective corneal epithelium, and that the staining pattern shown by adherent fluorescein correlates well with the staining pattern shown by rose bengal dye.
\end{abstract}

Since its introduction into ophthalmology by Pflüger ${ }^{1}$ in 1882 fluorescein has been widely used to detect corneal epithelial defects. An exhaustive review of its property of fluorescence and its use in ophthalmology has been published elsewhere. ${ }^{2}$ The dye is believed to penetrate into damaged epithelial cells or intercellular junctions but not to stain the cells themselves. ${ }^{23}$ The present study shows, however, that fluorescein also has an ability to adhere to and so to stain damaged corneal epithelial cells. This nonfluorescent phenomenon has thus far been clearly observed only in severe cases of herpes zoster and of keratoconjunctivitis sicca (KCS) keratopathy.

\section{Materials and methods}

A drop of commercially available $1 \%$ or $2 \%$ solution of fluorescein sodium (without preservative) was instilled into the temporal lower conjunctival sac in patients with various diseases of the ocular surface (herpes zoster, herpes simplex, KCS, epithelial oedema, epithelial erosions). The patients were encouraged to blink several times before and between photographs, which were taken by non-contact photomicrography, a method described elsewhere. ${ }^{4}$ After a series of photographs a drop of $1 \%$ rose bengal (without preservative) was added, and an attempt was made to capture identical locations on the cornea. The slides, taken with Ektachrome 100 or 200 ASA film, were examined and compared under high magnification (200$400 \times)$.

\section{Results}

In 12 patients with severe KCS and in four patients with severe herpes zoster keratopathy fluorescein adherence to the damaged epithelial surface was both recorded and correlated with rose bengal staining. It always appeared early

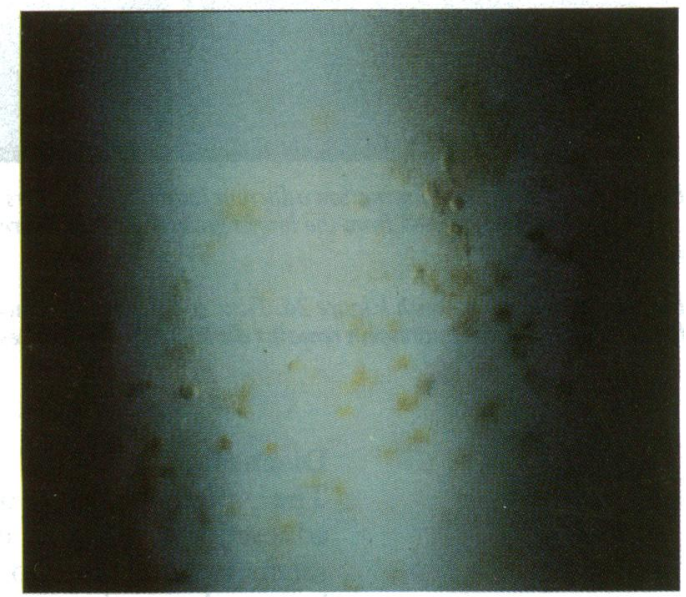

Figure 1 On retroillumination the brightly yellow hue of adherent fluorescein cannot be distinguished from dissolved fluorescein pooling in a cyst or in an epithelial defect (KCS, $1 \%$ fluorescein, retroillumination, $60 \times$ ).

after the application of the dye, and its hue varied with the mode of illumination. On retroillumination adherent fluorescein appeared brightly yellow (Fig 1) and could thus be confused with a thicker layer of dissolved fluorescein (for example when pooling in a cyst or in an epithelial defect). On focal illumination, however, the darker yellow or slightly brown hue shown by adherent fluorescein was easily distinguished from the brightly green fluorescent property of dissolved fluorescein (Figs 2a and 3a). When blue cobalt filter and focal illumination were used to enhance visualisation of fluorescence, adherent fluorescein appeared dark (Fig 2b).

The staining pattern of adherent fluorescein closely resembled that of rose bengal. That both staining patterns were basically identical became apparent by comparison of photomicrographs capturing the same area after fluorescein staining (Fig 3a) and after a subsequent rose bengal staining (Fig 3b). Indeed, rose bengal did not disclose any greater extent of damage. Some of the semi- or entirely devitalised elements indicated by adherent fluorescein were highlighted by the deep red hue of rose bengal, others stained more weakly, the resultant hue being a mixture of both. In a few the visibility of weak rose bengal staining was either precluded by the darker fluorescein background or absent. Comparison of photomicrographs also indicated that the heaviest staining by both dyes probably corresponded to the state of devitalisation: one of the stained elements disappeared between photographs, another changed position (Figs 3a and $3 b$ ). 

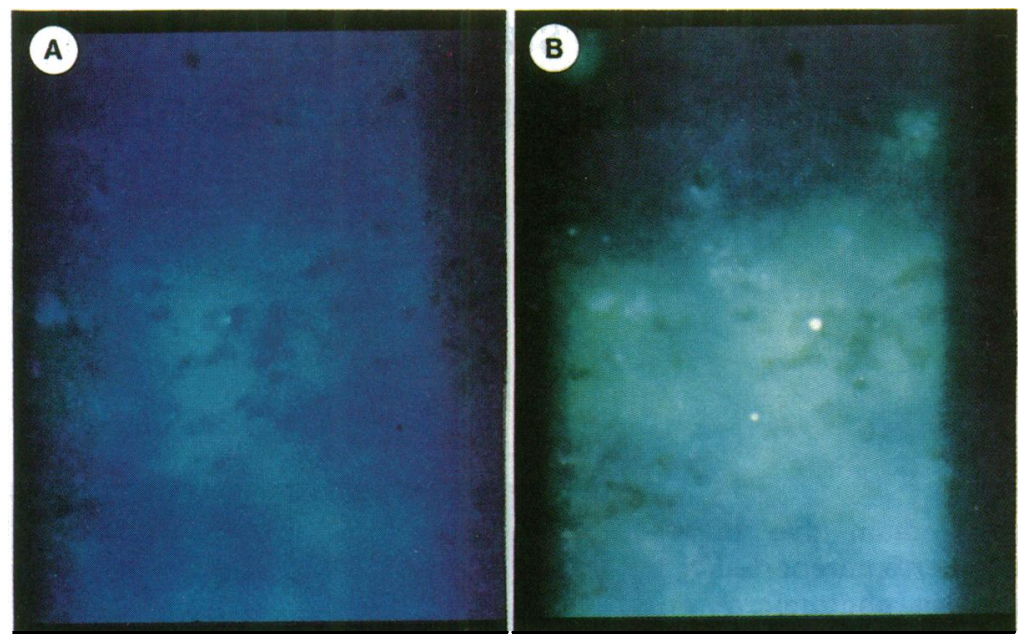

Figure $2 a$ On focal illumination adherent fluorescein appears darker, and the stained spots can easily be distinguished from the bright green dissolved fluorescein penetrating into the epithelium.

Figure $2 b$ Compare with Figure 2a. Blue cobalt filter enhances the visibility of dissolved fluorescein; adherent fluorescein remains dark (KCS, $1 \%$ fluorescein, focal illumination, $60 \times$ ).

\section{Discussion}

The phenomenon of fluorescein sodium adherence to diseased epithelial corneal surface seems to have hitherto escaped detection in slit lamp observations. Sequential photomicrographs have shown that it is present, and that its visibility depends on the intensity of staining and on the mode of illumination, a property basically shared with other coloured objects. On focal illumination its yellow-brown hue, similar to the hue of fluorescein sodium in the dry state (for example fluorescein impregnated paper) or in a concentrated solution, can easily be distinguished from the well known brightly green fluorescent property of fluorescein dissolved in the tear fluid or penetrating into epithelial defects and cysts.

In contrast to rose bengal, which is believed to stain dead or damaged cells, ${ }^{35}$ fluorescein is not believed to stain structures, even though both dyes are chemically similar, rose bengal being a derivative of fluorescein. The present study shows, however, that fixation of fluorescein occurs in a manner similar to that of rose bengal. As with rose bengal the exact nature of fluorescein bonds to the damaged cells remains unknown; in the cases presented here, both dyes seem to share a property of adherence to some devitalised, possibly keratinised elements. In other diseased states of the corneal epithelium which show rose bengal staining the absence of visible fluorescein adherence might either be real, showing differences in the chemical properties of the two dyes, or apparent, due to limitations of the observation method.

The detection of fluorescein adherence in $\mathrm{KCS}$ and herpes zoster keratopathies suggests
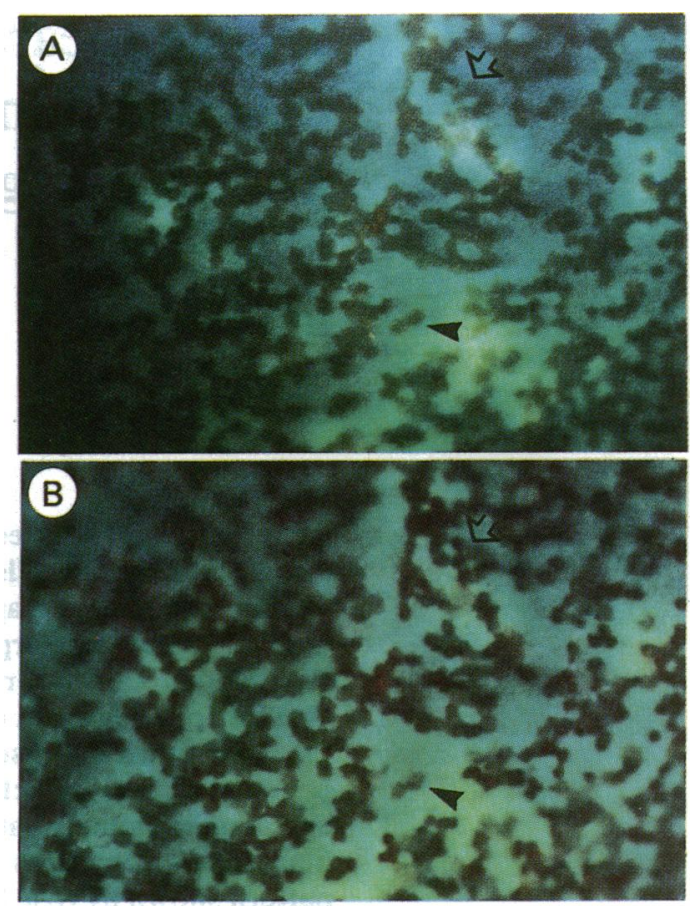

Figure $3 a$ Diseased elements on the corneal surface stained with adherent fluorescein show a brown-yellow hue of varying density; dissolved fluorescein appears bright green. For comparison the same spots as in Figure $3 b$ are indicated by arrows.

Figure $3 b$ Compare with Figure 3a. Subsequent staining with rose bengal shows basically the same pattern. In the original photomicrographs it is clearly visible that some of the diseased elements are highlighted by a deep red hue, others stain weakly, the hue corresponding to a mixture of both dyes; in a few no rose bengal staining is visible (arrowhead). One of the stained elements has disappeared and another one has changed position in the area indicated by open arrow (herpes zoster, $3 a-2 \%$ fluorescein, $3 b-1 \%$ rose bengal, focal illumination, $45 \times$ ). (Original magnification in the slides $24 \times$.)

that in these cases, when compared with rose bengal, fluorescein sodium is not only as good as but perhaps a more sensitive indicator of the extent of corneal surface damage. While rose bengal highlights only devitalised elements on the surface, fluorescein shows two phenomena simultaneously: fluid penetration into defective epithelium is highlighted by its fluorescence, and devitalised surface elements by its adherence.

This study was supported in part by a grant from Herman Järnhardts Stiftelse.

1 Pflüger. Zur Ernährung der Cornea. Klin Monatsbl Augenheilkd $1882 ; 69-81$

2 Romanchuk KG. Fluorescein: physicochemical factors affecting its fluorescence. Surv Ophthalmol 1982; 26: 269-83.

3 Havener WH. Ocular pharmacology, 4th ed. St Louis: Mosby, 1978: 413.

4 Tabery HM, Holm OC. Photography in vivo of epithelial lesions in the human cornea with non-contact high magnifilesions in the human cornea with non-contact high mag
cation technique. Acta Ophthalmol $(K b h), 1987 ; 65: 513$.

5 Norn MS. Vital staining of cornea and conjunctiva. Acta Ophthalmol (Kbh) 1972; 113 (suppl): 20. 